\title{
Late Diagnosis of Early Disseminated Lyme Disease: Perplexing Symptoms in a Gardener
}

\author{
Brooke E. Salzman, MD, Amber Stonehouse, MD, and James Studdiford, MD
}

The timely diagnosis of early disseminated Lyme disease presenting as multiple secondary erythema migrans lesions is sometimes delayed because this stage is infrequently encountered in a general practice. We report a case of a 60 -year-old woman whose initial complaints of an erythematous, "burning" rash and flu-like symptoms led to several laboratory tests with no specific diagnosis. The correct diagnosis was only made after sorting through other possibilities in the differential diagnosis. By reproducing the medical images and reviewing the medical literature, we underscore the importance of including Lyme disease in the list of diagnoses pertaining to diffuse skin rashes in the febrile patient. ( $\mathrm{J}$ Am Board Fam Med 2008;21:234-236.)

\section{Case Report}

A 60-year-old woman presented to her primary care doctor in June with a 1-day history of numerous, oval, diffusely erythematous patches scattered across her chest, abdomen, back, and extremities sparing her palms and soles (see Figures 1 and 2). The rash, which became more prominent after a hot shower or exposure to a warm, ambient environment, was also described as "burning" and "hot." Further, she reported a 1-week history of flu-like symptoms consisting of headache, neck pain, generalized body aches, fever, chills, and malaise. She also complained that her cheeks felt unusually hot and were bright red. She denied having any associated respiratory or gastrointestinal symptoms.

The patient denied a history of a recent tick or insect bite. However, on further questioning, she described herself as an avid gardener. She lives in a rural area of New Jersey where deer sightings are frequent.

The patient's bilateral facial erythema, which was reminiscent of the classic "slapped cheeks" appearance of parvovirus B-19 prompted serologic

This article was externally peer reviewed.

Submitted 24 August 2007; revised 20 November 2007; accepted 26 November 2007.

From the Department of Family and Community Medicine (BES, AS, JS), Division of Geriatrics (BES), Thomas Jefferson University, Philadelphia, PA.

Funding: none.

Conflict of interest: none declared.

Corresponding author: Brooke E. Salzman, MD, Department of Family and Community Medicine, Division of Geriatrics, Thomas Jefferson University, Philadelphia, PA 19107 (E-mail: brooke.salzman@jefferson.edu). testing for this viral agent. A complete blood count and a comprehensive metabolic panel were also performed. The results were all normal except the liver function tests, which were mildly elevated with an alanine aminotransferase of $75 \mathrm{U} / \mathrm{L}$, aspartate aminotransferase of $54 \mathrm{U} / \mathrm{L}$, and alkaline phosphatase of $148 \mathrm{U} / \mathrm{L}$. At a follow-up visit 4 days later, because of the persistence of numerous erythematous plaques accompanied by fever, myalgias, and arthralgias, Lyme disease (LD) was suspected and the patient was screened for Borrelia burgdorferi antibody with the standard enzyme-linked immunosorbent assay. This was positive with a reported value of $>5$ ( $\geq 1.10$ is considered positive). Western blot analysis for $\operatorname{IgM}$ was positive and negative for IgG, thus confirming acute, early disseminated LD. The patient was treated with $100 \mathrm{mg}$ of doxycycline twice daily for a period of 21 days with resolution of her symptoms. On further monitoring, her liver function tests returned to normal.

\section{Discussion}

This case describes a patient with secondary erythema migrans (EM) associated with early disseminated LD. The vast majority ( $75 \%$ to $80 \%$ ) of patients in the United States who present with early LD have only a single, primary EM lesion which occurs at the site of a tick bite. ${ }^{1}$ Primary EM lesions typically develop 7 to 10 days after a tick bite, with a reported range of 1 to 32 days. ${ }^{2}$ They are rapidly expanding, round or oval erythematous lesions that measure $>5 \mathrm{~cm}$ in largest diameter, and are flat or 


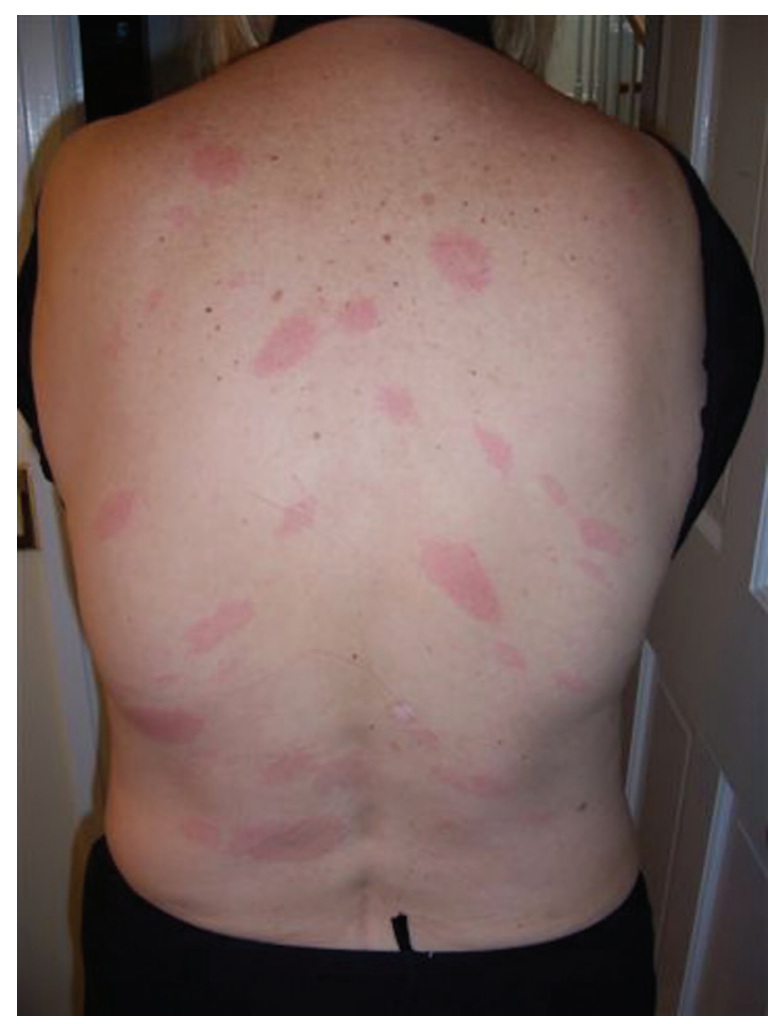

Figure 1. Numerous oval, erythematous patches scattered across this patient's chest, abdomen, back, and extremities sparing her palms and soles.

slightly raised. Early, untreated localized infection is often followed days to weeks later by hematogenous dissemination of the infection to multiple sites, which may include the skin ( $20 \%$ to $25 \%)$, the nervous system $(10 \%)$, the heart $(5 \%)$, or the joints $(60 \%))^{3}$

Secondary EM lesions of early disseminated LD arise at sites distant from the tick bite, indicating systemic dissemination of the spirochete Borrelia

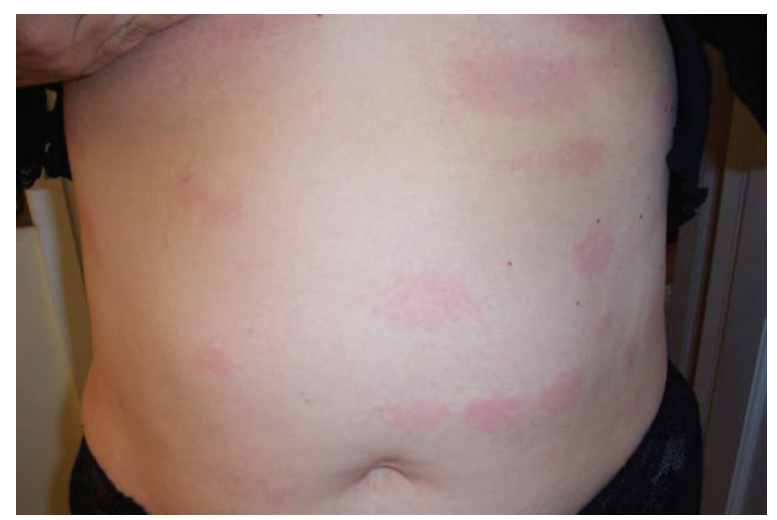

Figure 2. Anterior view. burgdorferi via blood or lymph. As such, secondary lesions lack the punctum indicative of a tick bite, which can often be found in primary EM lesions. The number of secondary lesions can range from 2 to $>30$, with a median of 14 , often appearing in 1 or 2 crops of similarly sized and shaped lesions. ${ }^{4-6}$ Secondary EM lesions are generally lighter, smaller, less edematous, and have less central clearing compared with primary EMs. ${ }^{4,5}$ Typically they first become apparent or intensify with elevations of body temperature. Patients with secondary EM without an obvious primary lesion may lack a host immune response to the bacterial antigen. ${ }^{4,5} \mathrm{Sec}-$ ondary EM lesions can appear on the head, face, trunk, and extremities, usually sparing the palms, soles, and mucus membranes.

Both early localized and early disseminated LD are usually accompanied by nonspecific, flu-like symptoms including myalgias, arthralgias, fatigue, headache, neck stiffness, and sometimes fever. ${ }^{1,3}$ Patients with secondary EM may have more constitutional symptoms than those with primary EM lesions alone. ${ }^{5}$ Upper respiratory tract and gastrointestinal symptoms are typically absent. ${ }^{1}$ The differential diagnosis for a diffuse, patchy, erythematous rash associated with flu-like symptoms can include urticaria, secondary syphilis, parvovirus B-19, lichen planus, erythema multiforme, pityriasis rosea, Lyme disease, and fixed drug eruption. Urticaria would probably not be associated with myalgias and arthralgias, as seen in our patient. Our patient's rash spared the palms and soles, unlike syphilis. The rash associated with parvovirus B-19 generally is limited to the cheeks. Erythema multiforme usually is described as iris or target-like vesicular lesions and typically involves the palms and soles. Our patient did not have the fine scaling papules and plaques with collarettes typically seen with pityriasis rosea.

Health care practitioners located in areas of endemicity for LD should especially become familiar with these early clinical manifestations. These geographic regions in the United States include areas of the Northeast (Massachusetts to Maryland), upper Midwest (Wisconsin, Minnesota), northern California, and Oregon. Most cases of LD occur during the late spring and summer, peaking in June and July. ${ }^{7}$ This time frame correlates with the nymphal stage of the life cycle of the Ixodes tick, which accounts for the large majority of LD transmission. $^{7}$ 
The diagnosis of early LD in the acute phase is generally a clinical one, based primarily on clinical findings, including the presence of a characteristic solitary EM and a history of tick exposure in an endemic area. Because of the high rate of falsenegative results in the acute phase of LD $(60 \%)$, routine use of serologic tests in patients with $\mathrm{EM}$ is not recommended. ${ }^{3}$ Such patients should be diagnosed and treated for LD without further testing. ${ }^{7,8}$ Cases in which serologic testing may provide valuable information include patients, such as ours, with clinical findings suggestive of early disseminated LD or patients with prolonged constitutional symptoms ( $>2$ weeks) suggestive of early LD without EM. ${ }^{8,9}$ When testing is indicated, both acutephase and convalescent-phase ( 2 weeks after the acute phase) serum samples should be tested using a 2-tiered assay recommended by the Centers for Disease Control. With the 2-tiered assay, a positive or indeterminate screening test (ie, enzyme-linked immunosorbent assay) is followed by a more specific confirmation test (ie, Western blot).

Additional testing that may be ordered on patients such as ours may include hepatic function panels. In the case of LD these tests (specifically transaminase determinations) are nonspecific measures of liver inflammation and not indicative of specific liver pathology. Such liver function test abnormalities have been reported to occur in $66 \%$ of patients with early disseminated LD. These are most often asymptomatic and resolve after 3 weeks of appropriate antibiotic therapy. ${ }^{10}$

The recommended treatment for adult patients with early localized or early disseminated LD (in the absence of specific neurologic manifestations including radiculopathy, cranial neuropathy, mononeuropathy multiplex, meningitis, or encephalomyelitis, or advanced atrioventricular heart block) is doxycycline (100 $\mathrm{mg}$ twice per day), amoxicillin (500 mg 3 times per day), or cefuroxime axetil (500 $\mathrm{mg}$ twice per day) for approximately 14 days. $^{8}$

\section{Conclusion}

It is important to consider Lyme disease (LD) in patients who present with unexplained flu-like symptoms during summer months, particularly in areas endemic for LD. Inquire about outdoor activities and routes of exposure when LD may be suspected. Clinicians should be aware that early LD may present with a diffuse rash indicating disseminated infection.

\section{References}

1. Steere AC, Sikand VK. The presenting manifestations of Lyme disease and the outcomes of treatment. N Engl J Med 2003;348:2472-4.

2. Smith RP, Schoen RT, Rahn DW, et al. Clinical characteristics and treatment outcome of early Lyme disease in patients with microbiologically confirmed erythema migrans. Ann Intern Med 2002;136:421-8.

3. Wormser GP. Early Lyme disease. N Engl J Med 29;354:2794-801.

4. McGinley-Smith DE, Tsao SS. Dermatoses from ticks. J Am Acad Dermatol 2003;49:363-92.

5. Melski JW, Reed KD, Mitchell PD, Barth GD. Primary and secondary erythema migrans in central Wisconsin. Arch Dermatol 1993;129:709-16.

6. Malane MS, Grant-Kels JM, Feder HM, Luger SW. Diagnosis of Lyme disease based on dermatologic manifestations. Ann Intern Med 1991;114:490-8.

7. Centers for Disease Control and Prevention. Lyme disease-United States, 2001-2002. Available athttp:// www.cdc.gov/mmwr/preview/mmwrhtml/mm5317a4. htm. Accessed March 7, 2007.

8. Wormser GP, Dattwyler RJ, Shapiro ED, et al. The clinical assessment, treatment, and prevention of Lyme disease, human granulocytic anaplasmosis, and babesiosis: Clinical practice guidelines by the Infectious Diseases Society of America. Clin Infect Dis 2006;43:1089-134.

9. Foy AJ Jr, Studdiford JS III. Lyme disease. Clinics Fam Pract 2005;7:191-208.

10. Horowitz HW, Dworkin B, Forseter G, et al. Liver function in early Lyme disease. Hepatology 1996;23: $1412-7$. 